\title{
Transverse momentum dependent forward neutron single spin asymmetries in transversely polarized $p+p$ collisions at $\sqrt{s}=200 \mathrm{GeV}$
}

U. A. Acharya,${ }^{20}$ C. Aidala, ${ }^{40}$ Y. Akiba,${ }^{53,54, \dagger}$ M. Alfred, ${ }^{22}$ V. Andrieux,${ }^{40}$ N. Apadula, ${ }^{27}$ H. Asano, ${ }^{33,53}$ B. Azmoun, ${ }^{7}$ V. Babintsev, ${ }^{23}$ N. S. Bandara, ${ }^{39}$ K. N. Barish, ${ }^{8}$ S. Bathe, ${ }^{5,54}$ A. Bazilevsky, ${ }^{7}$ M. Beaumier, ${ }^{8}$ R. Belmont, ${ }^{11,46}$ A. Berdnikov, ${ }^{56}$ Y. Berdnikov, ${ }^{56}$ L. Bichon, ${ }^{63}$ B. Blankenship, ${ }^{63}$ D. S. Blau, ${ }^{32,43}$ J. S. Bok ${ }^{45}$ V. Borisov, ${ }^{56}$ M. L. Brooks, ${ }^{35}$ J. Bryslawskyj, V. Bumazhnov, ${ }^{23}$ S. Campbell, ${ }^{12}$ V. Canoa Roman, ${ }^{59}$ R. Cervantes ${ }^{59}$ C. Y. Chi, ${ }^{12}$ M. Chiu, ${ }^{7}$ I. J. Choi, ${ }^{24}$ J. B. Choi, ${ }^{29, *}$ Z. Citron, ${ }^{64}$ M. Connors, ${ }^{20,54}$ R. Corliss, ${ }^{59}$ N. Cronin, ${ }^{59}$ M. Csanád, ${ }^{15}$ T. Csörgő, ${ }^{16,65}$ T. W. Danley, ${ }^{47}$ M. S. Daugherity, ${ }^{1}$ G. David, ${ }^{7,59}$ K. DeBlasio, ${ }^{44}$ K. Dehmelt, ${ }^{59}$ A. Denisov, ${ }^{23}$ A. Deshpande, ${ }^{54,59}$ E. J. Desmond, ${ }^{7}$ A. Dion, ${ }^{59}$ D. Dixit, ${ }^{59}$ J. H. Do, ${ }^{66}$ A. Drees,${ }^{59}$ K. A. Drees, ${ }^{6}$ J. M. Durham ${ }^{35}$ A. Durum, ${ }^{23}$ A. Enokizono, ${ }^{53,55}$ H. En'yo, ${ }^{53}$ R. Esha, ${ }^{59}$ S. Esumi, ${ }^{62}$ B. Fadem ${ }^{41}$ W. Fan ${ }^{59}$ N. Feege,${ }^{59}$ D. E. Fields, ${ }^{44}$ M. Finger, ${ }^{9}$ M. Finger, Jr., ${ }^{9}$ D. Firak, ${ }^{14}$ D. Fitzgerald, ${ }^{40}$ S. L. Fokin, ${ }^{32}$ J. E. Frantz, ${ }^{47}$ A. Franz, ${ }^{7}$ A. D. Frawley, ${ }^{19}$ Y. Fukuda, ${ }^{62}$ C. Gal,${ }^{59}$ P. Gallus,${ }^{13}$ P. Garg, ${ }^{3,59}$ H. Ge,${ }^{59}$ M. Giles,${ }^{59}$ F. Giordano, ${ }^{24}$ Y. Goto,${ }^{53,54}$ N. Grau, ${ }^{2}$ S. V. Greene ${ }^{63}$ M. Grosse Perdekamp, ${ }^{24}$ T. Gunji ${ }^{10}$ H. Guragain, ${ }^{20}$ T. Hachiya,${ }^{42,53,54}$

J. S. Haggerty, ${ }^{7}$ K. I. Hahn, ${ }^{17}$ H. Hamagaki, ${ }^{10}$ H. F. Hamilton, ${ }^{1}$ S. Y. Han, ${ }^{17,31}$ J. Hanks, ${ }^{59}$ S. Hasegawa, ${ }^{28}$ T. O. S. Haseler,${ }^{20}$ X. He, ${ }^{20}$ T. K. Hemmick, ${ }^{59}$ J. C. Hill, ${ }^{27}$ K. Hill, ${ }^{11}$ A. Hodges, ${ }^{20}$ R. S. Hollis, ${ }^{8}$ K. Homma, ${ }^{21}$ B. Hong, ${ }^{31}$ T. Hoshino, ${ }^{21}$ N. Hotvedt, ${ }^{27}$ J. Huang, ${ }^{7}$ S. Huang, ${ }^{63}$ K. Imai, ${ }^{28}$ M. Inaba ${ }^{62}$ A. Iordanova, ${ }^{8}$ D. Isenhower, ${ }^{1}$ D. Ivanishchev,${ }^{51}$ B. V. Jacak ${ }^{59}$ M. Jezghani, ${ }^{20}$ Z. Ji ${ }^{59}$ X. Jiang, ${ }^{35}$ B. M. Johnson $\odot,{ }^{7,20}$ D. Jouan, ${ }^{49}$ D. S. Jumper, ${ }^{24}$ J. H. Kang ${ }^{66}$ D. Kapukchyan, ${ }^{8}$ S. Karthas,${ }^{59}$ D. Kawall, ${ }^{39}$ A. V. Kazantsev, ${ }^{32}$ V. Khachatryan, ${ }^{59}$ A. Khanzadeev, ${ }^{51}$ A. Khatiwada, ${ }^{35}$ C. Kim, ${ }^{8,31}$ E.-J. Kim ${ }^{29}$ M. Kim,${ }^{57}$ D. Kincses, ${ }^{15}$ A. Kingan, ${ }^{59}$ E. Kistenev, ${ }^{7}$ J. Klatsky, ${ }^{19}$ P. Kline,${ }^{59}$ T. Koblesky, ${ }^{11}$ D. Kotov,${ }^{51,56}$ S. Kudo, ${ }^{62}$

B. Kurgyis, ${ }^{15}$ K. Kurita,${ }^{55}$ Y. Kwon, ${ }^{66}$ J. G. Lajoie, ${ }^{27}$ D. Larionova ${ }^{56}$ M. Larionova ${ }^{56}$ A. Lebedev, ${ }^{27}$ S. Lee, ${ }^{66}$

S. H. Lee ${ }^{27,40,59}$ M. J. Leitch, ${ }^{35}$ Y. H. Leung, ${ }^{59}$ N. A. Lewis, ${ }^{40}$ X. Li,${ }^{35}$ S. H. Lim,${ }^{35,52,66}$ M. X. Liu, ${ }^{35}$ V.-R. Loggins, ${ }^{24}$ S. Lökös, ${ }^{15}$ K. Lovasz,${ }^{14}$ D. Lynch, ${ }^{7}$ T. Majoros, ${ }^{14}$ Y. I. Makdisi, ${ }^{6}$ M. Makek, ${ }^{67}$ V. I. Manko, ${ }^{32}$ E. Mannel,,${ }^{7}$ M. McCumber,${ }^{35}$ P. L. McGaughey, ${ }^{35}$ D. McGlinchey, ${ }^{11,35}$ C. McKinney, ${ }^{24}$ M. Mendoza, ${ }^{8}$ W. J. Metzger, ${ }^{16}$ A. C. Mignerey, ${ }^{38}$ A. Milov ${ }^{64}$

D. K. Mishra, ${ }^{4}$ J. T. Mitchell, ${ }^{7}$ Iu. Mitrankov, ${ }^{56}$ G. Mitsuka,${ }^{30,54}$ S. Miyasaka, ${ }^{53,61}$ S. Mizuno ${ }^{53,62}$ M. M. Mondal,${ }^{59}$

P. Montuenga, ${ }^{24}$ T. Moon, ${ }^{31,66}$ D. P. Morrison, ${ }^{7}$ S. I. Morrow ${ }^{63}$ B. Mulilo, ${ }^{31,53}$ T. Murakami, ${ }^{33,53}$ J. Murata,${ }^{53,55}$ K. Nagai, ${ }^{61}$ K. Nagashima, ${ }^{21}$ T. Nagashima, ${ }^{55}$ J. L. Nagle, ${ }^{11}$ M. I. Nagy, ${ }^{15}$ I. Nakagawa,${ }^{53,54}$ K. Nakano, ${ }^{53,61}$ C. Nattrass, ${ }^{60}$ S. Nelson, ${ }^{18}$ T. Niida, ${ }^{62}$ R. Nouicer, ${ }^{7,54}$ T. Novák, ${ }^{16,65}$ N. Novitzky, ${ }^{59,62}$ A. S. Nyanin, ${ }^{32}$ E. O’Brien, ${ }^{7}$ C. A. Ogilvie, ${ }^{27}$

J. D. Orjuela Koop, ${ }^{11}$ J. D. Osborn, ${ }^{40,48}$ A. Oskarsson, ${ }^{36}$ G. J. Ottino, ${ }^{44}$ K. Ozawa, ${ }^{30,62}$ V. Pantuev, ${ }^{25}$ V. Papavassiliou, ${ }^{45}$ J. S. Park, ${ }^{57}$ S. Park, ${ }^{53,57,59}$ S. F. Pate, ${ }^{45}$ M. Patel, ${ }^{27}$ W. Peng, ${ }^{63}$ D. V. Perepelitsa ${ }^{7,11}$ G. D. N. Perera,${ }^{45}$ D. Yu. Peressounko, ${ }^{32}$ C. E. PerezLara, ${ }^{59}$ J. Perry, ${ }^{27}$ R. Petti, ${ }^{7}$ M. Phipps, ${ }^{7,24}$ C. Pinkenburg, ${ }^{7}$ R. P. Pisani, ${ }^{7}$ M. Potekhin, ${ }^{7}$ A. Pun, ${ }^{47}$

M. L. Purschke, ${ }^{7}$ P. V. Radzevich,${ }^{56}$ N. Ramasubramanian, ${ }^{59}$ K. F. Read ${ }^{48,60}$ D. Reynolds, ${ }^{58}$ V. Riabov,${ }^{43,51}$ Y. Riabov, ${ }^{51,56}$ D. Richford, ${ }^{5}$ T. Rinn, ${ }^{24,27}$ S. D. Rolnick, ${ }^{8}$ M. Rosati, ${ }^{27}$ Z. Rowan, ${ }^{5}$ J. Runchey, ${ }^{27}$ A. S. Safonov, ${ }^{56}$ T. Sakaguchi ${ }^{7}$ H. Sako, ${ }^{28}$ V. Samsonov, ${ }^{43,51}$ M. Sarsour, ${ }^{20}$ S. Sato, ${ }^{28}$ B. Schaefer ${ }^{63}$ B. K. Schmoll ${ }^{60}$ K. Sedgwick, ${ }^{8}$ R. Seidl,${ }^{53,54}$ A. Sen, ${ }^{27,60}$ R. Seto, ${ }^{8}$ A. Sexton, ${ }^{38}$ D. Sharma, ${ }^{59}$ D. Sharma, ${ }^{59}$ I. Shein ${ }^{23}$ T.-A. Shibata, ${ }^{53,61}$ K. Shigaki, ${ }^{21}$ M. Shimomura, ${ }^{27,42}$ T. Shioya, ${ }^{62}$ P. Shukla, ${ }^{4}$ A. Sickles, ${ }^{24}$ C. L. Silva ${ }^{35}$ D. Silvermyr,${ }^{36}$ B. K. Singh, ${ }^{3}$ C. P. Singh, ${ }^{3}$ V. Singh, ${ }^{3}$ M. Slunečka, ${ }^{9}$ K. L. Smith, ${ }^{19}$ M. Snowball, ${ }^{35}$ R. A. Soltz, ${ }^{34}$ W. E. Sondheim, ${ }^{35}$ S. P. Sorensen, ${ }^{60}$ I. V. Sourikova, ${ }^{7}$ P. W. Stankus, ${ }^{48}$ S. P. Stoll, ${ }^{7}$

T. Sugitate, ${ }^{21}$ A. Sukhanov, ${ }^{7}$ T. Sumita, ${ }^{53}$ J. Sun, ${ }^{59}$ X. Sun, ${ }^{20}$ Z. Sun, ${ }^{14}$ J. Sziklai, ${ }^{65}$ K. Tanida ${ }^{28,54,57}$ M. J. Tannenbaum, ${ }^{7}$ S. Tarafdar, ${ }^{63,64}$ G. Tarnai, ${ }^{14}$ R. Tieulent, ${ }^{20,37}$ A. Timilsina, ${ }^{27}$ T. Todoroki, ${ }^{54,62}$ M. Tomášek, ${ }^{13}$ C. L. Towell, ${ }^{1}$ R. S. Towell, ${ }^{1}$ I. Tserruya, ${ }^{64}$ Y. Ueda, ${ }^{21}$ B. Ujvari, ${ }^{14}$ H. W. van Hecke, ${ }^{35}$ J. Velkovska, ${ }^{63}$ M. Virius, ${ }^{13}$ V. Vrba, ${ }^{13,26}$ N. Vukman, ${ }^{67}$

X. R. Wang, ${ }^{45,54}$ Y. S. Watanabe, ${ }^{10}$ C. P. Wong, ${ }^{20,35}$ C. L. Woody, ${ }^{7}$ Y. Wu, ${ }^{8}$ C. Xu, ${ }^{45}$ Q. Xu, ${ }^{63}$ L. Xue, ${ }^{20}$ S. Yalcin, ${ }^{59}$ Y. L. Yamaguchi, ${ }^{59}$ H. Yamamoto, ${ }^{62}$ A. Yanovich, ${ }^{23}$ J. H. Yoo, ${ }^{31}$ I. Yoon, ${ }^{57}$ H. Yu, ${ }^{45,50}$ I. E. Yushmanov, ${ }^{32}$ W. A. Zajc, ${ }^{12}$ A. Zelenski, ${ }^{6}$ Y. Zhai, ${ }^{27}$ S. Zharko, ${ }^{56}$ and L. Zou ${ }^{8}$

\section{(PHENIX Collaboration)}

\author{
${ }^{1}$ Abilene Christian University, Abilene, Texas 79699, USA \\ ${ }^{2}$ Department of Physics, Augustana University, Sioux Falls, South Dakota 57197, USA \\ ${ }^{3}$ Department of Physics, Banaras Hindu University, Varanasi 221005, India \\ ${ }^{4}$ Bhabha Atomic Research Centre, Bombay 400 085, India \\ ${ }^{5}$ Baruch College, City University of New York, New York, New York 10010, USA \\ ${ }^{6}$ Collider-Accelerator Department, Brookhaven National Laboratory, Upton, New York 11973-5000, USA \\ ${ }^{7}$ Physics Department, Brookhaven National Laboratory, Upton, New York 11973-5000, USA \\ ${ }^{8}$ University of California-Riverside, Riverside, California 92521, USA \\ ${ }^{9}$ Charles University, Ovocný trh 5, Praha 1, 11636 Prague, Czech Republic
}


${ }^{10}$ Center for Nuclear Study, Graduate School of Science, University of Tokyo, 7-3-1 Hongo, Bunkyo, Tokyo 113-0033, Japan

${ }^{11}$ University of Colorado, Boulder, Colorado 80309, USA

${ }^{12}$ Columbia University, New York, New York 10027 and Nevis Laboratories, Irvington, New York 10533, USA

${ }^{13}$ Czech Technical University, Zikova 4, 16636 Prague 6, Czech Republic

${ }^{14}$ Debrecen University, H-4010 Debrecen, Egyetem tér 1, Hungary

${ }^{15}$ ELTE, Eötvös Loránd University, H-1117 Budapest, Pázmány P. s. 1/A, Hungary

${ }^{16}$ Esz,terházy Károly University, Károly Róbert Campus, H-3200 Gyöngyös, Mátrai út 36, Hungary

${ }^{17}$ Ewha Womans University, Seoul 120-750, Korea

${ }^{18}$ Florida A\&M University, Tallahassee, Florida 32307, USA

${ }^{19}$ Florida State University, Tallahassee, Florida 32306, USA

${ }^{20}$ Georgia State University, Atlanta, Georgia 30303, USA

${ }^{21}$ Hiroshima University, Kagamiyama, Higashi-Hiroshima 739-8526, Japan

${ }^{22}$ Department of Physics and Astronomy, Howard University, Washington, D.C. 20059, USA

${ }^{23}$ IHEP Protvino, State Research Center of Russian Federation, Institute for High Energy Physics, Protvino, 142281, Russia

${ }^{24}$ University of Illinois at Urbana-Champaign, Urbana, Illinois 61801, USA

${ }^{25}$ Institute for Nuclear Research of the Russian Academy of Sciences, prospekt 60-letiya Oktyabrya 7a, Moscow 117312, Russia

${ }^{26}$ Institute of Physics, Academy of Sciences of the Czech Republic, Na Slovance 2, 18221 Prague 8, Czech Republic

${ }^{27}$ Iowa State University, Ames, Iowa 50011, USA

${ }^{28}$ Advanced Science Research Center, Japan Atomic Energy Agency, 2-4 Shirakata Shirane,

Tokai-mura, Naka-gun, Ibaraki-ken 319-1195, Japan

${ }^{29}$ Jeonbuk National University, Jeonju 54896, Korea

${ }^{30}$ KEK, High Energy Accelerator Research Organization, Tsukuba, Ibaraki 305-0801, Japan

${ }^{31}$ Korea University, Seoul 02841, Korea

${ }^{32}$ National Research Center “Kurchatov Institute," Moscow 123098, Russia

${ }^{33}$ Kyoto University, Kyoto 606-8502, Japan

${ }^{34}$ Lawrence Livermore National Laboratory, Livermore, California 94550, USA

${ }^{35}$ Los Alamos National Laboratory, Los Alamos, New Mexico 87545, USA

${ }^{36}$ Department of Physics, Lund University, Box 118, SE-221 00 Lund, Sweden

${ }^{37}$ IPNL, CNRS/IN2P3, Univ Lyon, Universit Lyon 1, F-69622 Villeurbanne, France

${ }^{38}$ University of Maryland, College Park, Maryland 20742, USA

${ }^{39}$ Department of Physics, University of Massachusetts, Amherst, Massachusetts 01003-9337, USA

${ }^{40}$ Department of Physics, University of Michigan, Ann Arbor, Michigan 48109-1040, USA

${ }^{41}$ Muhlenberg College, Allentown, Pennsylvania 18104-5586, USA

${ }^{42}$ Nara Women's University, Kita-uoya Nishi-machi Nara 630-8506, Japan

${ }^{43}$ National Research Nuclear University, MEPhI, Moscow Engineering Physics Institute, Moscow 115409, Russia

${ }^{44}$ University of New Mexico, Albuquerque, New Mexico 87131, USA

${ }^{45}$ New Mexico State University, Las Cruces, New Mexico 88003, USA

${ }^{46}$ Physics and Astronomy Department, University of North Carolina at Greensboro, Greensboro, North Carolina 27412, USA

${ }^{47}$ Department of Physics and Astronomy, Ohio University, Athens, Ohio 45701, USA

${ }^{48}$ Oak Ridge National Laboratory, Oak Ridge, Tennessee 37831, USA

${ }^{49} I P N-O r s a y$, Univ. Paris-Sud, CNRS/IN2P3, Université Paris-Saclay, BP1, F-91406 Orsay, France

${ }^{50}$ Peking University, Beijing 100871, People's Republic of China

${ }^{51}$ PNPI, Petersburg Nuclear Physics Institute, Gatchina, Leningrad region 188300, Russia

${ }^{52}$ Pusan National University, Pusan 46241, Korea

${ }^{53}$ RIKEN Nishina Center for Accelerator-Based Science, Wako, Saitama 351-0198, Japan

${ }^{54}$ RIKEN BNL Research Center, Brookhaven National Laboratory, Upton, New York 11973-5000, USA

${ }^{55}$ Physics Department, Rikkyo University, 3-34-1 Nishi-Ikebukuro, Toshima, Tokyo 171-8501, Japan

${ }^{56}$ Saint Petersburg State Polytechnic University, St. Petersburg 195251, Russia

${ }^{57}$ Department of Physics and Astronomy, Seoul National University, Seoul 151-742, Korea

${ }^{58}$ Chemistry Department, Stony Brook University, SUNY, Stony Brook, New York 11794-3400, USA 


\author{
${ }^{59}$ Department of Physics and Astronomy, Stony Brook University, \\ SUNY, Stony Brook, New York 11794-3800, USA \\ ${ }^{60}$ University of Tennessee, Knoxville, Tennessee 37996, USA \\ ${ }^{61}$ Department of Physics, Tokyo Institute of Technology, Oh-okayama, Meguro, Tokyo 152-8551, Japan \\ ${ }^{62}$ Tomonaga Center for the History of the Universe, University of Tsukuba, Tsukuba, Ibaraki 305, Japan \\ ${ }^{63}$ Vanderbilt University, Nashville, Tennessee 37235, USA \\ ${ }^{64}$ Weizmann Institute, Rehovot 76100, Israel \\ ${ }^{65}$ Institute for Particle and Nuclear Physics, Wigner Research Centre for Physics, Hungarian Academy of \\ Sciences (Wigner RCP, RMKI) H-1525 Budapest 114, P.O. Box 49, Budapest, Hungary \\ ${ }^{66}$ Yonsei University, IPAP, Seoul 120-749, Korea \\ ${ }^{67}$ Department of Physics, Faculty of Science, University of Zagreb, \\ Bijenička c. 32 HR-10002 Zagreb, Croatia
}

(Received 30 November 2020; accepted 21 January 2021; published 26 February 2021)

\begin{abstract}
In 2015, the PHENIX collaboration has measured very forward $(\eta>6.8)$ single spin asymmetries of inclusive neutrons in transversely polarized proton-proton and proton-nucleus collisions at a center of mass energy of $200 \mathrm{GeV}$. A previous publication from this dataset concentrated on the nuclear dependence of such asymmetries. In this measurement the explicit transverse momentum dependence of inclusive neutron single spin asymmetries for proton-proton collisions is extracted using a bootstrapping unfolding technique on the transverse momenta. This explicit transverse momentum dependence will help improve the understanding of the mechanisms that create these asymmetries.
\end{abstract}

DOI: 10.1103/PhysRevD.103.032007

\section{INTRODUCTION}

At the beginning of the era of polarized proton collisions at the relativistic heavy ion collider (RHIC), a dedicated experiment based on a prototype zero-degree calorimeter (ZDC) [1] was set up to initially study very forward neutral pion asymmetries in transversely polarized proton collisions in relation to earlier results that showed nonzero results [2]. Instead of finding a neutral pion asymmetry, which was only recently discovered at low transverse momentum by the RHICf experiment [3], a sizable neutron asymmetry was found in the forward direction of the transversely polarized proton beam [4].

Earlier theoretical studies related very forward neutron production to the one-pion-exchange (OPE) model [5-7] in which the exchange of one pion between the proton and another colliding particle can create the outgoing neutron. Such a model was reasonably successful in describing unpolarized, very forward neutron production as previously observed at the ISR [8]. However, a simple pion exchange model would not be able to describe any spin dependence of the observed neutron distributions. To accommodate that, an interference with another particle exchange would

\footnotetext{
*Deceased.

†Spokesperson: akiba@rcf.rhic.bnl.gov
}

Published by the American Physical Society under the terms of the Creative Commons Attribution 4.0 International license. Further distribution of this work must maintain attribution to the author(s) and the published article's title, journal citation, and DOI. Funded by SCOAP ${ }^{3}$. be necessary to have helicity-flip and nonflip amplitudes available that can create a single spin left-right asymmetry. Within the general framework of Regge theory [9], such an interference could be accomplished when adding also a scalar meson exchange and the resulting pseudoscalarscalar meson interference would then create the asymmetry. Recent calculations of such an OPE based description of very forward neutron single spin asymmetries [10] are able to qualitatively describe the RHIC measurements which, by now, include transversely polarized proton-proton collisions at $\sqrt{s}=62,200$, as well as $500 \mathrm{GeV}$ [11], although the transverse momentum information enters only indirectly via the different collision energies.

The very different asymmetries observed in protonnucleus collisions [12], with different sign and much larger magnitude, indicate that at high impact parameters and at least for high- $Z$ nuclei ultraperipheral collisions (UPC) [13] also contribute to these asymmetries in a very different way. These data together with the recent very forward nonzero neutral pion result [3] may provide crucial information to the underlying mechanisms that create these asymmetries.

So far, none of these results have been extracted with an explicit transverse momentum dependence while the different collision energies provide some indirect information on it. Obtaining it can directly test the proposed mechanism and the dependence that results from its theory calculation [10]. Therefore, extracting the actual transverse momentum dependence is the focus of this publication. A substantial understanding is required of the transverse momentum smearing in the PHENIX ZDCs. Also needed are determinations of systematic uncertainties in unfolding transverse 
momenta, which were studied via a Monte Carlo (MC) bootstrap method as described later.

In the following sections, the detector description, analyzed datasets and the forward neutron selection are covered. Next are described the procedure for unfolding the neutron single spin asymmetries as a function of the reconstructed transverse momenta to obtain the true transverse momentum dependence. Then, the final results are presented before summarizing.

\section{DATASETS}

In 2015, the PHENIX experiment recorded polarized proton-proton and proton-nucleus collision data at a center of mass energy of $200 \mathrm{GeV}$. In the proton-proton collision data, the beams were transversely polarized with the spin direction pointing vertically up or down with respect to the plane defined by the accelerator ring.

Inclusive neutrons were detected with the ZDC, which comprise three modules of $\mathrm{Cu}-\mathrm{W}$ alloy absorbers layered with optical fibers of 1.7 nuclear interaction lengths each (51 radiation lengths per module), covering a projected area of $10 \mathrm{~cm}$ by $10 \mathrm{~cm}$ transverse to the beam direction. The absorber layers of the ZDC are tilted 45 degrees upward to maximize the light yield from Čerenkov light. The location of the ZDCs is $18 \mathrm{~m}$ up and downstream of the PHENIX beam interaction point, thus covering a range of pseudorapidity $\eta>6.8$. The ZDC is used to measure the energy of forward neutrons, and its energy resolution is about $20 \%$ for neutron energies of $100 \mathrm{GeV}$. Between the first and the second module, approximately at the position of the maximal hadronic shower are located scintillator strip detectors with a projected width of $15 \mathrm{~mm}$ horizontally and vertically. This shower max detector (SMD) is used to determine the position of the neutrons that are selected by calculating the weighted average of the deposited energy for all strip positions. The position resolution of the SMD for neutrons is $\approx 1 \mathrm{~cm}$. Additionally, the SMD is used for local polarimetry of the polarized beams by making use of the nonzero neutron asymmetries in proton-proton collisions and allowed to track the transverse spin orientation or confirm the spin orientation to be rotated in the longitudinal direction. See Ref. [14] for a more detailed detector description.

Collision events were selected for this result by a logical OR of north and south ZDCs that require approximately an energy deposit of more than $15 \mathrm{GeV}$ on either detector. Within the proton collisions were accumulated about $35 \mathrm{M}$ neutron events that were triggered by the ZDCs.

\section{EVENT AND PARTICLE SELECTION CRITERIA}

Neutron candidates in the north ZDC were selected by requiring more than $3 \%$ of the total deposited energy to be in the second ZDC module. This effectively rejects photon candidates, that deposited their energy in the first module due to being electromagnetic showers. Also nonzero hits in both horizontal and vertical SMDs are required to reliably estimate the neutron position and transverse momentum. Furthermore, the reconstructed neutron energies were selected between 40 and $120 \mathrm{GeV}$. The hit position as defined by the SMDs has to be within 0.5 to $4 \mathrm{~cm}$ in radius from the nominal beam position. Additionally, data under stable running conditions with no problems in the polarized beam diagnostics were selected. The transverse momentum $P_{T}$ is reconstructed from the neutron energy $E$, the radius of the average hit position $r$ and the distance from the interaction point $z_{\mathrm{ZDC}}$ :

$$
P_{T}=\frac{r}{z_{\mathrm{ZDC}}} E
$$

The events that fulfill the above conditions are then binned in four transverse momentum bins of [0.01, 0.06, 0.11, 0.16, $0.21] \mathrm{GeV} / c$ and six equidistant azimuthal angular bins that cover full azimuth around the polarized beam direction. The two spin states are kept separated for the unfolding, but to obtain the asymmetries needed in the bootstrap MC method, they are also directly calculated here as

$$
A_{N}(\phi)=\frac{1}{\langle P\rangle} \frac{N^{+}(\phi)-\mathcal{R} N^{-}(\phi)}{N^{+}(\phi)+\mathcal{R} N^{-}(\phi)},
$$

where $\langle P\rangle$ is the average beam polarization (for this running period 52\% [15]) and $N^{ \pm}$are the yields of neutrons in the up/ down spin state as a function of azimuthal angle $\phi$ that is defined relative to the spin-up direction. $\mathcal{R}$ is the ratio of accumulated luminosities for the down and up spin states, and is close to unity in this analysis. The actual $A_{N}$ is then calculated by fitting a sine modulation to it with magnitude and phase as free parameters.

As systematic uncertainties, the amount of charged particle background (dominated by protons) and the uncertainty of the beam center position need to be evaluated. Unlike other years, no charge veto counter in front of the ZDC was installed in this running period. This resulted in a rather asymmetric charged hadron background predominantly from protons that are swept into the ZDC by the dipole magnet which joins and separates the two beams. The fraction of charged hadron background was statistically subtracted on the spin dependent yield level by applying the background fractions that were obtained in the 2008 running period when the charge veto counter was installed. The statistical uncertainties on these background fractions were then assigned as systematic uncertainties on the resulting raw asymmetries.

The central beam position relative to the ZDC also cannot be perfectly determined due to the large lever arm as well as varying beam conditions. As such, the assigned beam position was artificially varied by $1 \mathrm{~cm}$ horizontally and $0.5 \mathrm{~cm}$ vertically around the nominal beam position, respectively. All neutron positions, transverse momenta, 
and azimuthal angles were recalculated before evaluating the asymmetries. These variations were motivated by the uncertainties based on two independent methods of reconstructing the beam center positions using the ZDCs. The combined uncertainties on the asymmetries from charged background and beam position are then used as a basis for variation of the bootstrap MC method of unfolding the asymmetries as discussed in the next section. The uncertainties due to charged background and beam position remain negligible compared to the large systematic uncertainties this unfolding introduces.

\section{TRANSVERSE MOMENTUM UNFOLDING}

As hadronic showers develop a substantial size and the segmentation of the SMDs is limited, the reconstructed neutron energy and in particular the transverse momentum are smeared. The transverse momentum dependence of the single spin asymmetries is however of much interest for the understanding of the mechanism that creates these asymmetries.

We have performed detailed MC simulations using five different types of event generators as input to full GEANT3 [16] simulations of the forward region of PHENIX $[17,18]$ including the ZDCs, SMDs, the beampipe as well as the dipole magnet that merges and separates the incoming and outgoing beams and is responsible for an asymmetric spray from charged particles. These GEANT simulations have been shown to describe these effects, as well as differences between top and bottom that originate from the light collection and back scattering in the top part of the ZDC. The composition, energy, and momentum distributions of particles in the far forward region are not very well understood in general, and therefore different types of generators were used to gauge the impact of these differences on the unfolded asymmetries. The three full generators PYTHIA6.1 [19], PYTHIA8.2 [20], and DPMJET3.1 [21] were applied, where in particular diffractive processes are handled very differently.

Additionally, an empirical distribution of forward neutrons in longitudinal and transverse momentum was used to mimic an OPE model. In this case, a pion that balances the energy and momentum between the incoming proton and the thrown neutron (i.e., $p \rightarrow \pi^{+}+n$ ) was collided with the other beam using PYTHIA8 again (i.e., $\pi+p$ ). Moreover, as the forward $p+A$ results have indicated [12], ultraperipheral collisions can also play a role in forward neutron production although that will be more prominent in proton-nucleus collisions. Therefore, the yield and distribution of photons from the other beam was simulated using STARLIGHT [22] and collided with the proton beam using PYTHIA8 again.

As none of these generators is intrinsically spin dependent, spin effects ( $w$ in the following expression) were simulated by reweighting generated events as a function of true transverse momentum and azimuthal angle where the spin state was randomly assigned. Three different functional forms were used in the reweighting to provide as much flexibility as possible for the true transverse momentum dependence of the single spin asymmetries. The most general parametrization is a third order polynomial in the transverse momentum with a vanishing constant term due to the requirement for the asymmetry to vanish at zero transverse momentum:

$w=\left(a \cdot P_{T, g}+b \cdot P_{T, g}^{2}+c \cdot P_{T, g}^{3}\right) \sin \left(\phi_{g}+\lambda \cdot \pi\right)$,

where $P_{T, g}$ and $\phi_{g}$ are the true transverse momenta and azimuthal angles, respectively, and $\lambda( \pm 1)$ is the spin state, while $a, b$, and $c$ are free parameters that are varied. A second functional form (with $a$ and $b>0$ free parameters) is based on a power-law behavior:

$$
w=\left(a \cdot P_{T, g}^{b}\right) \sin \left(\phi_{g}+\lambda \cdot \pi\right),
$$

and the last parametrization (with $a$ and $b$ free parameters) follows an exponential form that eventually reaches an asymptotic constant:

$$
w=a\left(1-\mathrm{e}^{b \cdot P_{T, g}}\right) \sin \left(\phi_{g}+\lambda \cdot \pi\right) .
$$

In the power law parametrization, only positive powers are allowed to avoid unphysical nonzero asymmetries at zero transverse momentum.

For each set of parameters, functional form, and MC generator, the single spin asymmetries were extracted from the reconstructed kinematic variables that included these weights based on the true variables.

In a first step, the reconstructed asymmetries that were obtained from the data are compared to the reconstructed asymmetries from MC for a large number of variations of the parameters. The quality of a set of parameters, functional form and MC generator in reproducing the data asymmetries was evaluated by calculating the $\chi^{2}$ between the actual data points and the smeared asymmetry points. While the MC statistics are generally large enough, many functional forms can describe the data within the experimentally measured uncertainties.

Figure 1 displays the functional forms probed for the OPE motivated MC generator. The regions where a parametrization results in a $\chi^{2}$ below 10 units is also visualized to highlight the range of reasonable parametrizations. Despite the different transverse momentum distributions of forward neutrons in the different MC generators, their best asymmetry distributions are very similar for each set of functional forms. In all functional forms, a rapidly rising asymmetry is preferred at small $P_{T}$, while at intermediate transverse momentum (within the second and third data points) no large variation in the asymmetries is visible. The exponential function generally prefers the asymptotic value to be reached already at these transverse momenta. 

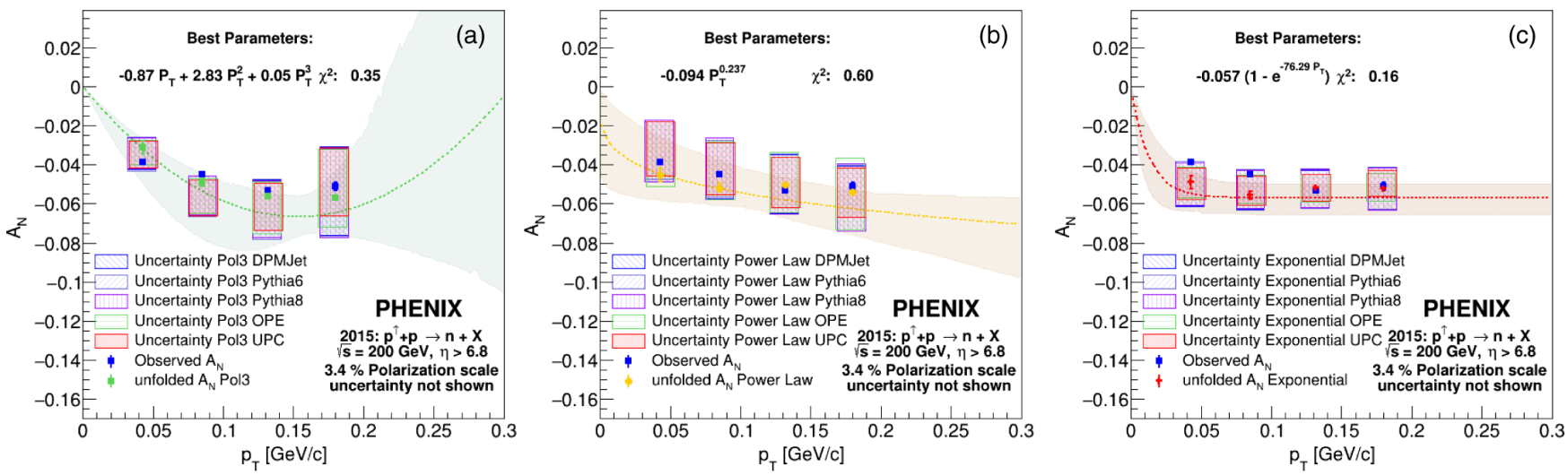

FIG. 1. True asymmetry parametrizations as a function of transverse momentum for (a) a third order polynomial dependence "Pol3," (b) a power-law dependence "Power Law," and (c) an exponential dependence "Exponential." The shaded regions represent the regions where the $\chi^{2}$ between the smeared asymmetries related to this parametrization and the asymmetries reconstructed from data (solid [blue] squares) is below 10 units. The dashed lines represent the best matching parametrizations. Also displayed are the unfolded asymmetries (a) solid [dark green] squares, (b) solid [orange] circles, and (c) solid [red] hyphens, as obtained from the best parametrizations of the OPE generator. The rms ranges of unfolded asymmetries are visualized as shaded boxes for the various MC generators.

At higher transverse momenta above $0.2 \mathrm{GeV} / c$, the sensitivity is very limited for all functional forms despite a nonzero smearing into the observed range. As such, the slightly rising, constant or even diminishing asymmetries at high transverse momenta can describe the measured data reasonably well.

In a second step, the spin-dependent data yields that are two-dimensional in reconstructed transverse momentum and azimuthal angle are unfolded using the spin-dependent, weighted smearing matrices obtained for each set of parameters, functional form and MC generator set. For the unfolding itself, the TSVDUnfold package of ROOT [23] based on a regularized singular value decomposition [24] was used. After the unfolding, the asymmetries are calculated from the unfolded yields as described above. The best parametrization for each functional form and MC generator is used to obtain the central point of the unfolded asymmetries and statistical uncertainties. The impact of the variation of parameters is evaluated by obtaining the root mean square (rms) of the spread of unfolded asymmetries which are weighted by the inverse of their respective $\chi^{2}$ to take the quality of a parameter set into account. These uncertainties are also displayed in Fig. 1, together with the unfolded asymmetries using the OPE generator.

The variation of the unfolded asymmetries is displayed in Fig. 2 for each transverse momentum bin and parametrization, while spreads from the different MC generators had been combined equally. The central values from these distributions have been taken as the final asymmetry values while the rms value is taken as the uncertainty due to the various parametrizations.

In addition to these uncertainties, further systematic uncertainties are studied by varying the regularization parameter in the TSVDUnfold method as well as the uncertainties due to the statistical uncertainties on the smearing matrices themselves. However, most of these values are within the boundaries of the uncertainties obtained from the variation of parameters and functional forms. Only those systematic contributions that exceed the aforementioned uncertainties have been added in quadrature.
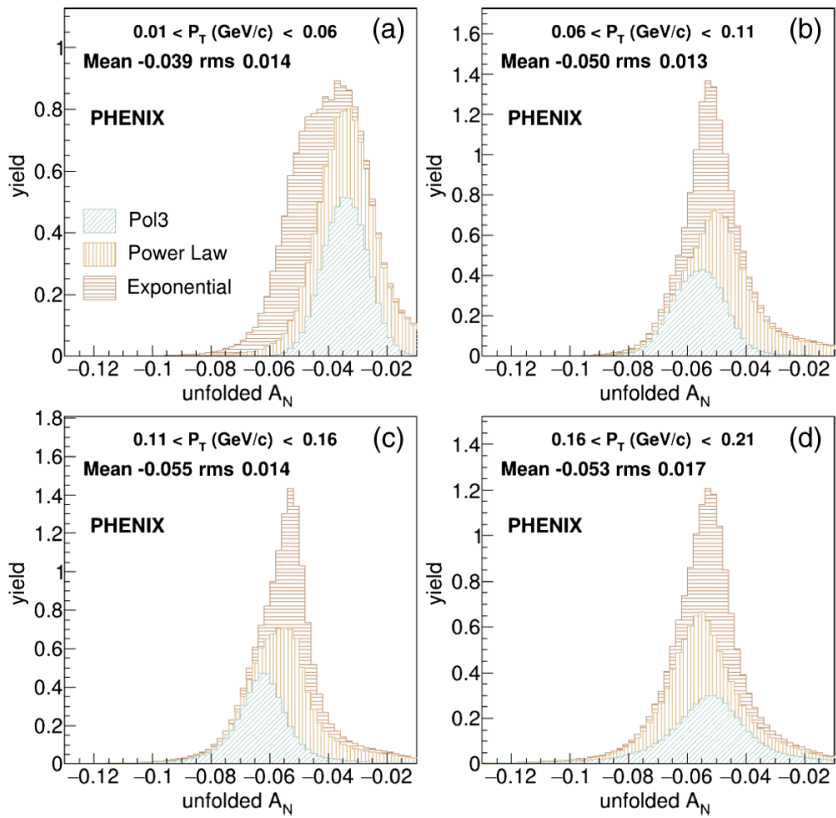

FIG. 2. Relative likelihood distributions of unfolded asymmetries for each transverse momentum bin for all sets of parameters of each functional form weighted by the inverse of its $\chi^{2}$. All different $\mathrm{MC}$ generators distributions have been combined in these panels. The distributions of the third order polynomial parametrization (shaded [light green] area "Pol3"), power law behavior (vertically hatched [light orange] area "Power Law") and exponential (horizontally hatched [light red] area "Exponential") have been stacked in these figures. The overall central and rms values are also displayed. 


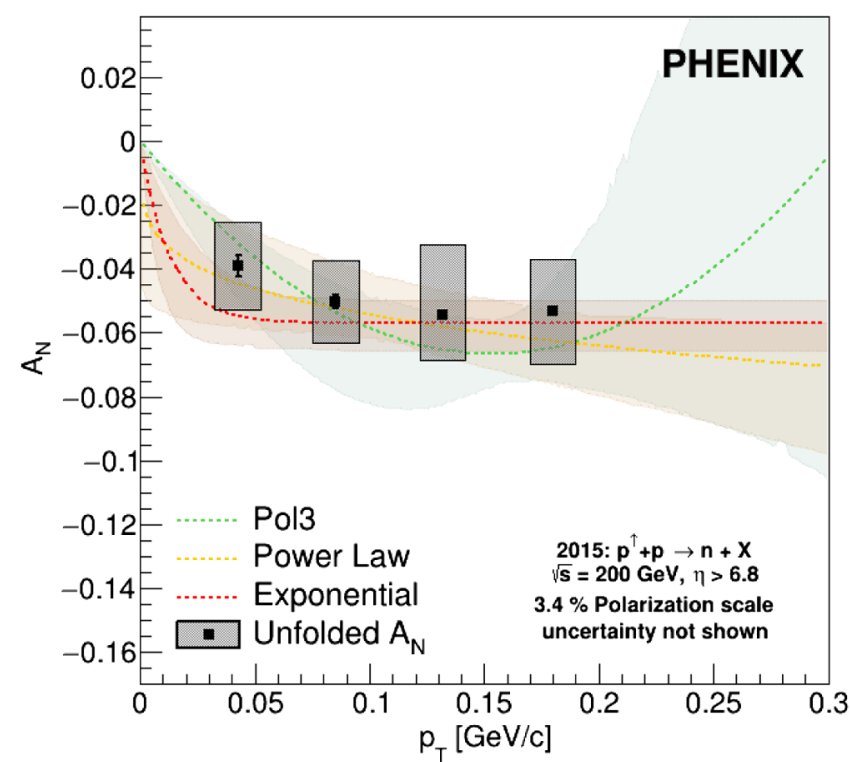

FIG. 3. Neutron transverse single spin asymmetries as a function of the true transverse momentum. The data points represent the unfolded asymmetries obtained via the average over all parametrizations and MC generators. The uncertainty boxes represent the systematic uncertainties due to the parametrization, functional form, MC generator, and unfolding procedure.

\section{RESULTS}

The inclusive neutron asymmetries obtained from the average of all parametrizations and $\mathrm{MC}$ generators are displayed in Fig. 3 as a function of the true transverse momentum. The final results are tabulated in Table I.

The absolute values of the asymmetries are consistent with an increase with transverse momentum but show an indication of leveling off at higher transverse momenta. A simple linear dependence as suggested by [10], as well as the central values of transverse momentum integrated asymmetries at different collision energies [4,18], seems not necessarily to be preferred by the data. However, a simple linear dependence cannot be excluded within uncertainties either. From the MC reweighting exercise no substantial differences between the different MC generators have been seen.

TABLE I. Neutron single spin asymmetries as a function of transverse momentum after unfolding transverse-momentum and azimuthal-angular smearing. $\Delta A_{N}$ corresponds to the statistical uncertainties while the last two columns specify the upper and lower systematic uncertainties $\delta A_{N}$.

\begin{tabular}{lcccc}
\hline \hline$\left\langle P_{T}\right\rangle(\mathrm{GeV} / c)$ & $A_{N}$ & $\Delta A_{N}$ & \multicolumn{2}{c}{$\delta A_{N}$} \\
\hline 0.043 & -0.039 & \pm 0.003 & +0.014 & -0.014 \\
0.085 & -0.050 & \pm 0.002 & +0.013 & -0.013 \\
0.132 & -0.055 & \pm 0.002 & +0.022 & -0.014 \\
0.180 & -0.053 & \pm 0.001 & +0.017 & -0.017 \\
\hline \hline
\end{tabular}

Taking into account the indication of very different asymmetries in ultraperipheral collisions in proton-nucleus collisions [12,13] and in particular a different sign, it appears that the UPC contribution to the proton-proton collisions is limited in this $p_{T}$ region. This is expected given the electromagnetic nature of the interaction being proportional with $Z^{2}$. However, in these inclusive results some contribution from UPC events may remain, which could alter the transverse momentum behavior in comparison to the purely hadronic theoretical calculations.

\section{SUMMARY}

In summary, the PHENIX experiment has measured for the first time the transverse momentum dependence of very forward neutron single spin asymmetries in protonproton collisions at a center of mass energy of $200 \mathrm{GeV}$. With these measurements the first reliable tests of the suggested mechanisms producing such forward neutron asymmetries can be performed. While the uncertainties from the unfolding are very sizable, a simple linear transverse momentum dependence as suggested in [10] is not inconsistent; however, the asymmetries appear to level off at higher transverse momenta. Instead, a much slower rise of the asymmetries or even a turnaround at larger transverse momenta is favored when considering the best parametrizations. To understand the mechanisms in even more detail, the correlations with other detector activity will be useful.

\section{ACKNOWLEDGMENTS}

We thank the staff of the Collider-Accelerator and Physics Departments at Brookhaven National Laboratory and the staff of the other PHENIX participating institutions for their vital contributions. We acknowledge support from the Office of Nuclear Physics in the Office of Science of the Department of Energy, the National Science Foundation, Abilene Christian University Research Council, Research Foundation of SUNY, and Dean of the College of Arts and Sciences, Vanderbilt University (USA); Ministry of Education, Culture, Sports, Science, and Technology and the Japan Society for the Promotion of Science (Japan); Conselho Nacional de Desenvolvimento Científico e Tecnológico and Fundação de Amparo à Pesquisa do Estado de São Paulo (Brazil); Natural Science Foundation of China (People's Republic of China); Croatian Science Foundation and Ministry of Science and Education (Croatia); Ministry of Education, Youth and Sports (Czech Republic); Centre National de la Recherche Scientifique, Commissariat à l'Énergie Atomique, and Institut National de Physique Nucléaire et de Physique des Particules (France); Bundesministerium für Bildung und Forschung, Deutscher Akademischer Austausch Dienst, and Alexander von Humboldt Stiftung (Germany); J. Bolyai Research Scholarship, EFOP, the New National Excellence Program (ÚNKP), NKFIH, and 
OTKA (Hungary); Department of Atomic Energy and Department of Science and Technology (India); Israel Science Foundation (Israel); Basic Science Research and SRC (CENuM) Programs through NRF funded by the Ministry of Education and the Ministry of Science and ICT (Korea); Physics Department, Lahore University of Management Sciences (Pakistan), Ministry of Education and
Science, Russian Academy of Sciences, Federal Agency of Atomic Energy (Russia); VR and Wallenberg Foundation (Sweden); the U.S. Civilian Research and Development Foundation for the Independent States of the Former Soviet Union; the Hungarian American Enterprise Scholarship Fund; the US-Hungarian Fulbright Foundation; and the US-Israel Binational Science Foundation.
[1] C. Adler, A. Denisov, E. Garcia, M. J. Murray, H. Strobele, and S. N. White, The RHIC zero degree calorimeter, Nucl. Instrum. Methods Phys. Res., Sect. A 470, 488 (2001).

[2] D. L. Adams et al. (E581 and E704 Collaborations), Comparison of spin asymmetries and cross-sections in pi0 production by $200-\mathrm{GeV}$ polarized anti-protons and protons, Phys. Lett. B 261, 201 (1991).

[3] M. H. Kim et al. (RHICf Collaboration), Transverse SingleSpin Asymmetry for Very Forward Neutral Pion Production in Polarized $p+p$ Collisions at $\sqrt{s}=510 \mathrm{GeV}$, Phys. Rev. Lett. 124, 252501 (2020).

[4] Y. Fukao et al., Single transverse-spin asymmetry in very forward and very backward neutral particle production for polarized proton collisions at $\sqrt{s}=200 \mathrm{GeV}$, Phys. Lett. B 650, 325 (2007).

[5] J. Soffer and N. A. Tornqvist, Origin of the Polarization for Inclusive $\Lambda$ Production in $p p$ Collisions, Phys. Rev. Lett. 68, 907 (1992).

[6] U. D'Alesio and H. J. Pirner, Target fragmentation in $p \mathrm{p}$, e p and gamma p collisions at high-energies, Eur. Phys. J. A 7, 109 (2000).

[7] B. Kopeliovich, B. Povh, and I. Potashnikova, Deep inelastic electroproduction of neutrons in the proton fragmentation region, Z. Phys. C 73, 125 (1996).

[8] W. Flauger and F. Monnig, Measurement of inclusive zeroangle neutron spectra at the CERN ISR, Nucl. Phys. B109, 347 (1976).

[9] P. D. B. Collins, An Introduction to Regge Theory and High-Energy Physics, Cambridge Monographs on Mathematical Physics (Cambridge University Press, Cambridge, England, 2009).

[10] B. Z. Kopeliovich, I. K. Potashnikova, Ivan Schmidt, and J. Soffer, Single transverse spin asymmetry of forward neutrons, Phys. Rev. D 84, 114012 (2011).

[11] A. Adare et al. (PHENIX Collaboration), Inclusive cross section and single transverse spin asymmetry for very forward neutron production in polarized $p+p$ collisions at $\sqrt{s}=200 \mathrm{GeV}$, Phys. Rev. D 88, 032006 (2013).

[12] C. Aidala et al. (PHENIX Collaboration), Nuclear Dependence of the Transverse-Single-Spin Asymmetry for Forward Neutron Production in Polarized $p+A$ Collisions at $\sqrt{s_{N N}}=200 \mathrm{GeV}$, Phys. Rev. Lett. 120, 022001 (2018).
[13] G. Mitsuka, Recently measured large $A_{N}$ for forward neutrons in $p^{\uparrow}+A$ collisions at $\sqrt{s_{N N}}=200 \mathrm{GeV}$ explained through simulations of ultraperipheral collisions and hadronic interactions, Phys. Rev. C 95, 044908 (2017).

[14] K. Adcox et al. (PHENIX Collaboration), PHENIX detector overview, Nucl. Instrum. Methods Phys. Res., Sect. A 499, 469 (2003).

[15] RHIC Polarimetry Group, RHIC polarization for Runs 9-12, RHIC/CAD accelerator physics note 490, (2013).

[16] R. Brun, F. Bruyant, F. Carminati, S. Giani, M. Maire, A. McPherson, G. Patrick, and L. Urban, GEANT detector description and simulation tool, CERN-W5013, CERNW-5013, W5013, W-5013 (1994).

[17] M. Togawa, Measurements of the leading neutron production in polarized $p p$ collision at $\sqrt{s}=200 \mathrm{GeV}$, Ph.D. thesis, Kyoto University, 2008.

[18] A. Adare et al. (PHENIX Collaboration), Measurement of transverse-single-spin asymmetries for midrapidity and forward-rapidity production of hadrons in polarized $p+p$ collisions at $\sqrt{s}=200$ and $62.4 \mathrm{GeV}$, Phys. Rev. D 90, 012006 (2014).

[19] T. Sjostrand, L. Lonnblad, and S. Mrenna, PYTHIA 6.2: Physics and Manual (2001), arXiv:hep-ph/0108264.

[20] T. Sjöstrand, S. Ask, J. R. Christiansen, R. Corke, N. Desai, P. Ilten, S. Mrenna, S. Prestel, C. O. Rasmussen, and P. Z. Skands, An introduction to PYTHIA8.2, Comput. Phys. Commun. 191, 159 (2015).

[21] S. Roesler, R. Engel, and J. Ranft, The Monte Carlo event generator DPMJET-III, in International Conference on Advanced Monte Carlo for Radiation Physics, Particle Transport Simulation and Applications (MC 2000) (2000), p. 1033, arXiv:hep-ph/0012252.

[22] S. R. Klein, J. Nystrand, J. Seger, Y. Gorbunov, and J. Butterworth, STARlight: A Monte Carlo simulation program for ultra-peripheral collisions of relativistic ions, Comput. Phys. Commun. 212, 258 (2017).

[23] R. Brun and F. Rademakers, ROOT: An object oriented data analysis framework, Nucl. Instrum. Methods Phys. Res., Sect. A 389, 81 (1997).

[24] A. Hocker and V. Kartvelishvili, SVD approach to data unfolding, Nucl. Instrum. Methods Phys. Res., Sect. A 372, 469 (1996). 